\title{
GASES EN SANGRE DE ARTERIA UMBILICAL DE NEONATOS A TÉRMINO EN ALTURA
}

\author{
Wilfredo Villamonte ${ }^{1,2, a}$, Darío Escalante ${ }^{2,3, b}$, Janet Yabar ${ }^{3, b}$, María Jerí1,4,c, \\ Paola Peralta ${ }^{4, c}$, Robert Ochoa ${ }^{4, d}$
}

RESUMEN

Con el objetivo de determinar los valores normales de gases en sangre en la arteria umbilical de neonatos a término a $3400 \mathrm{~m}$ de altitud se hizo un estudio transversal, realizado en la sangre de la arteria umbilical de 300 neonatos a término, adecuados para la edad gestacional y cuyo parto se dio entre enero de 2010 a diciembre de 2011 en el Hospital Nacional Adolfo Guevara Velazco de EsSalud (Cusco, Perú). Se encontró que la media del pH de neonatos sanos a término fue de 7,33 $\pm 0,07$, los valores para los percentiles 5 y 95 fueron 7,18 y 7,40 respectivamente. En conclusión, se han construido las tablas con los percentiles 5 al 95 para el pH, pO , $\mathrm{pCO}_{2}, \mathrm{SO}_{2}, \mathrm{p} 50$, exceso de bases y $\mathrm{HCO}_{3}$, de la arteria umbilical de neonatos a término a $3400 \mathrm{~m}$ de altitud.

Palabras clave: Valores de referencia; Sangre de cordón umbilical; Recién nacido; Altitud (fuente DeCS BIREME).

\section{UMBILICAL ARTERY BLOOD GASES OF TERM NEONATES AT ALTITUDE}

\begin{abstract}
In order to determine the normal values of arterial blood gases in the umbilical artery of term infants at $3400 \mathrm{~m}$ altitude, a cross-sectional study was conducted. It was performed in the umbilical artery blood of 300 term infants, with an adequate gestational age and whose birth took place between January 2010 and December 2011 at the Essalud National Hospital Adolfo Guevara Velazco (Cusco, Peru). It was found that the average $\mathrm{pH}$ of healthy term infants was $7.33 \pm 0.07$; the values for percentiles 5 and 95 were 7.18 and 7.40 respectively. Tables with the 5th and 95th percentiles for $\mathrm{pH}, \mathrm{pO} 2, \mathrm{pCO} 2, \mathrm{SO} 2, \mathrm{p} 50$, base excess and $\mathrm{HCO} 3$ of the umbilical artery of term infants at $3400 \mathrm{~m}$ altitude are provided.
\end{abstract}

Key words: Reference values; Fetal blood; Infant, newborn; Altitude (source: MeSH NLM).

\section{INTRODUCCIÓN}

Es conocido que la presión parcial de oxígeno $\left(\mathrm{pO}_{2}\right)$ disminuye conforme se asciende en altitud, se ha descrito respecto a este hecho que estaría asociado a variaciones en los valores normales de la fisiología del neonato, en particular a aquellas relacionadas con la antropometría del neonato ${ }^{(1,2)}$.

Asimismo, el incremento en la tasa metabólica basal de las gestantes, que residen por encima de los $2000 \mathrm{~m}$ de altitud, mediado por las variaciones en los niveles de progesterona y estrógeno incrementan la quimiosensibilidad en ellas a la hipoxia, situación que busca mantener durante su embarazo el contenido arterial de oxígeno en sangre en valores semejantes a los descritos en gestantes que residen a nivel del mar ${ }^{(3)}$. Es por ello que la hipoxia hipobárica que aparece a partir de los $2000 \mathrm{~m}$ de altitud produce que la gestante hiperventile $y$, consecuentemente, disminuya la presión parcial de dióxido de carbono $\left(\mathrm{pCO}_{2}\right)$ en su sangre. Ello se traduce en una alcalosis respiratoria más una acidosis metabólica compensatoria en la sangre materna ${ }^{(4)}$. Esta sangre materna es llevada a través del cordón umbilical al feto. 
El análisis de los gases arteriales en sangre de arteria umbilical ha demostrado ser útil en el seguimiento del neonato y es una medida inmediata para conocer el ambiente fetal intraparto y brinda la información más exacta para conocer su estado ácido-base ${ }^{(5)}$. Por lo que la evaluación de éste permite conocer el estado ácidobase fetal.

En el Perú, más de 9 millones de personas viven por encima de los $2000 \mathrm{~m}$ de altitud ${ }^{(6)}$. En el mundo son más de 140 millones de personas que tienen esta condición, razón por lo cual es importante conocer las características gasométricas de la sangre fetal en altura. Es por ello que el objetivo de este estudio fue determinar los valores normales de gases en sangre en la arteria umbilical de neonatos a término nacidos en altura.

\section{EL ESTUDIO}

Se realizó un estudio transversal, en el cual se incluyó a neonatos nacidos entre enero de 2010 a diciembre de 2011 en el Hospital Nacional Adolfo Guevara Velazco de EsSalud del Cusco, el cual se ubica a $3400 \mathrm{~m}$ de altitud. Se incluyó a los neonatos de gestantes a término (por fecha de última regla y ecografía), de gestaciones únicas, sin patologías, nacidos por parto vaginal, catalogados como adecuados para la edad gestacional (7) y que tuvieran un puntaje apgar $\geq 7$ puntos a los 5 minutos de haber nacido.

Para la toma de muestra se consideró lo siguiente: inmediatamente producido la expulsión fetal, se pinzó el cordón umbilical en su porción proximal y distal en relación a la placenta, con una separación de $10 \mathrm{~cm}$. De esta porción de cordón umbilical se obtuvo $2 \mathrm{~mL}$ de sangre ${ }^{(5)}$ con una jeringa con heparina (Marquest ${ }^{\mathrm{TM}}$ ), se cubrió la aguja con un tapón de goma y, posteriormente, se realizó la determinación de la gasometría con el analizador de gases Cobas $^{\circledR} 21$ (Roche Diagnostics, $\mathrm{GmHb}$, Alemania) con calibración previa a la medición, de acuerdo a los $3400 \mathrm{~m}$ de altitud, dentro de los 30 minutos siguientes.

Las variables evaluadas fueron: potencial de hidrógeno $(\mathrm{pH})$, presión parcial de oxígeno $\left(\mathrm{pO}_{2}\right)$, presión parcial de dióxido de carbono $\left(\mathrm{pCO}_{2}\right)$, grado de afinidad de la hemoglobina por el oxígeno $\left(\mathrm{P}_{50}\right)$, bicarbonato $\left(\mathrm{HCO}_{3}\right)$,saturación de oxígeno $\left(\mathrm{SO}_{2}\right)$ y exceso de bases (EB). Se calcularon medias, desviación estándar y los percentiles de las variables estudiadas. El Análisis de los resultados se realizó con el programa SPSS versión 17. Todas las madres dieron su consentimiento para formar parte del estudio.
Tabla 1. Características maternas de neonatos a término a $3400 \mathrm{~m}$ de altitud

\begin{tabular}{lccc}
\hline \multicolumn{1}{c}{ Características } & Media & DE & (IC 95\%) \\
\hline Edad & 31,2 & 5,8 & $(30,0-32,5)$ \\
Gestaciones & 2,4 & 1,5 & $(2,0-2,7)$ \\
Hemoglobina (g/dL) & 13,8 & 1,0 & $(13,6-14,0)$ \\
Número de hijos vivos & 0,9 & 1,1 & $(0,7-1,2)$ \\
Antecedentes de abortos previos & 0,4 & 0,8 & $(0,2-0,6)$ \\
\hline
\end{tabular}

DE: desviación estándar.

\section{HALLAZGOS}

Participaron del estudio 300 neonatos. La edad materna promedio fue de $31,2 \pm 8,8$ años, quienes tuvieron en promedio $2,4 \pm 1,5$ gestaciones previas, otras características se detallan en la Tabla 1. En relación a los neonatos, el promedio de la edad gestacional encontrado fue de 39,4 $\pm 1,1$ semanas, en tanto que la media de peso al nacer fue de 3248,6 \pm 281,8 gramos, otras características de ellos se muestran en la Tabla 2.

Se encontró que en sangre de cordón umbilical de neonatos nacidos a $3400 \mathrm{~m}$ de altitud la media del $\mathrm{pH}$ fue de 7,33 \pm 0,07; además, los valores encontrados para los percentiles 5 y 95 fueron 7,18 y 7,40 respectivamente. Del mismo modo, la media de la $\mathrm{pCO}_{2}$ fue 42,22 $\pm 9,46$, los valores encontrados para los percentiles 5 y 95 fueron 32,70 y 53,99 , respectivamente. Para el caso del $\mathrm{HCO}_{3}$, la media encontrada fue de $21,74 \pm 2,10$; y los valores de los percentiles 5 y 95 fueron 17,42 y 24,39 , respectivamente (Tabla 3 ).

Tabla 2. Características de neonatos a término a $3400 \mathrm{~m}$ de altitud

\begin{tabular}{lrcc}
\hline \multicolumn{1}{c}{ Características } & Media & DE & (IC 95\%) \\
\hline Semana gestacional & 39,4 & 1,1 & $(39,2-39,7)$ \\
\hline Peso $(\mathrm{g})$ & 3248,6 & 281,8 & $(3186,0-3311,0)$ \\
Talla $(\mathrm{cm})$ & 49,8 & 1,3 & $(49,5-50,1)$ \\
\hline Índice ponderal & 2,6 & 0,2 & $(2,6-2,7)$ \\
\hline Perímetro cefálico $(\mathrm{cm})$ & 34,2 & 1,2 & $(34,0-34,5)$ \\
Perímetro torácico $(\mathrm{cm})$ & 33,4 & 1,3 & $(33,1-33,7)$ \\
Hematocrito de arteria & 48 & 5,6 & $(46,7-49,2)$ \\
umbilical (\%) & 673,2 & 97,9 & $(651,4-695,0)$ \\
Peso placenta (g) & 4,9 & 0,7 & $(4,8-5,1)$ \\
\hline Índice peso fetal/placentario & & &
\end{tabular}

DE: desviación estándar 
Tabla 3. Media y percentiles de gases arteriales en arteria umbilical en neonatos a término a $3400 \mathrm{~m}$ de altitud

\begin{tabular}{|c|c|c|c|c|c|c|c|c|c|c|}
\hline \multirow{2}{*}{ Variable } & \multirow{2}{*}{ Media } & \multirow{2}{*}{ DE } & \multirow{2}{*}{ (IC 95\%) } & \multicolumn{7}{|c|}{ Percentiles } \\
\hline & & & & 5 & 10 & 25 & 50 & 75 & 90 & 95 \\
\hline $\mathrm{pH}$ & 7,33 & 0,07 & $(7,31-7,35)$ & 7,18 & 7,22 & 7,28 & 7,32 & 7,36 & 7,39 & 7,40 \\
\hline $\mathrm{pO}_{2}(\mathrm{mmHg})$ & 14,89 & 6,34 & $(13,40-16,60)$ & 7,09 & 8,88 & 11,70 & 14,89 & 17,70 & 20,12 & 22,11 \\
\hline $\mathrm{pCO}_{2}(\mathrm{mmHg})$ & 42,22 & 9,46 & $(39,90-44,60)$ & 32,70 & 34,59 & 37,60 & 42,22 & 46,73 & 51,17 & 53,99 \\
\hline $\mathrm{SO}_{2}$ & 25,90 & 13,06 & $(22,60-29,20)$ & 6,70 & 7,91 & 13,00 & 25,90 & 32,23 & 40,69 & 44,19 \\
\hline$P_{50}$ & 19,69 & 3,42 & $(18,80-20,50)$ & 12,48 & 16,85 & 18,53 & 19,69 & 21,40 & 22,75 & 23,53 \\
\hline EB (mmol/L) & $-4,95$ & 2,88 & $(-5,30--4,60)$ & $-10,29$ & $-8,77$ & $-6,53$ & $-4,95$ & $-3,30$ & $-2,13$ & $-1,40$ \\
\hline $\mathrm{HCO}_{3}(\mathrm{mmol} / \mathrm{L})$ & 21,74 & 2,10 & $(21,20-22,30)$ & 17,42 & 18,20 & 19,38 & 21,74 & 22,50 & 23,60 & 24,39 \\
\hline
\end{tabular}

$\mathrm{DE}$ : desviación estándar, $\mathrm{PH}$ :potencial de hidrogeno, $\mathrm{pO}_{2}$ : presión parcial de oxígeno, $\mathrm{pCO}_{2}$ : presión parcial de dióxido de carbono, $\mathrm{SO}_{2}$ : saturación de Oxígeno, $\mathrm{P}_{50}$ : grado de afinidad de la hemoglobina por el oxígeno, EB: exceso de bases y $\mathrm{HCO}_{3}$ : bicarbonato

\section{DISCUSIÓN}

Se ha descrito que el $\mathrm{pH}$ de la sangre de la arteria umbilical a nivel del mar, disminuye conforme avanza la edad gestacional, de 7,28 en los neonatos de gestaciones pretérmino a 7,24 en los neonatos de gestantes a término ${ }^{(11)}$. Ello sería la traducción de un mejor intercambio gaseoso en los primeros ${ }^{(8)}$, el cual se correlaciona con un mayor flujo en la vena umbilical por unidad de masa fetal ${ }^{(9)}$. En nuestro estudio se encontró un $\mathrm{pH}$ de 7,33 en recién nacidos de gestaciones a término en altura, que refleja un estado menos ácido (10), el cual es semejante a los neonatos pretérminos nacidos a nivel del mar. Ello mostraría un mejor intercambio gaseoso por parte de la placenta, ya que permite desviar a la izquierda la curva de disociación de la hemoglobina y así liberar más oxígeno a los tejidos ${ }^{(11)}$.

En nuestro estudio se encontró que la $\mathrm{pO}_{2}$ en muestras de sangre de la arteria umbilical de neonatos a $3400 \mathrm{~m}$ de altitud es baja $(14,9 \mathrm{mmHg})$ en comparación con lo descrito en la literatura a nivel del mar ${ }^{(7,8,10)}$ y a $1800 \mathrm{~m}$ de altitud en Estados Unidos de Norteamérica ${ }^{(10)}$ que podría relacionarse con una mayor extracción de oxígeno de la vena umbilical por parte del feto que nace en altura ${ }^{(12)}$. Asimismo, la $\mathrm{pCO}_{2}$ encontrada en estos neonatos fue de $42,2 \mathrm{mmHg}$, la cual es menor a lo descrito a nivel del mar ${ }^{(7,8,10)}$. Cifra que incrementa el contenido de oxígeno de la hemoglobina ${ }^{(11)}$. A nivel del mar se han descrito valores de $E B$ de 4 a $-4.3 \mathrm{mmol} / \mathrm{L}^{(7,8,10)}$, en nuestro estudio, hallamos un valor menor de EB $(-4.9 \mathrm{mmol} / \mathrm{L})$, valor que es incluso menor a los $-3,3 \mathrm{mmol} / \mathrm{L}$ descritos a $1800 \mathrm{~m}$ de altitud ${ }^{(7)}$, ello podría deberse a un mayor consumo de bases.

Algunas limitaciones deben ser reconocidas. Previamente se ha descrito que la pobreza podría influenciar sobre el crecimiento fetal (13-15), en nuestro estudio no se ha controlado ese factor, por cuanto se desconoce su efecto sobre nuestro análisis. La descripción de la gasometría en la arteria umbilical de una población socioeconómicamente intermedia específicamente a $3400 \mathrm{~m}$ de altitud en el Perú, hace que nuestro estudio no sea fácilmente comparable con otros en la misma línea de investigación. Sin embargo, el presente estudio es uno de los primeros en describir las variaciones en la gasometría en neonatos sanos nacidos en altura.

En conclusión se han construido las tablas con los percentiles 5 al 95 para el $\mathrm{pH}, \mathrm{pO}_{2}, \mathrm{pCO}_{2}, \mathrm{SO}_{2}, \mathrm{p} 50$, EB y $\mathrm{HCO}_{3}$, de muestras de sangre de arteria umbilical de neonatos sanos a término a $3400 \mathrm{~m}$ de altitud.

Contribuciones de autoría: WV y MJ han participado en la concepción, diseño, recolección, análisis de datos, análisis estadístico y redacción del artículo. DE y JY participaron en la revisión del artículo. PP y RO realizaron la recolección. Todos aprobaron la versión final a publicar.

Fuentes de financiamiento: autofinanciado.

Conflicto de interés: los autores declaran no tener conflictos de interés. 


\section{REFERENCIAS BIBLIOGRÁFICAS}

1. Mortola JP, Frappell PB, Agüero L, Armstrong. Birth weight and altitude: A study in peruvian communities. J Pediatr. 2000;136(3):324-9.

2. Villamonte W, Jerí M, Lajo L, Monteagudo Y, Diez G. Peso al Nacer en recién nacidos a término en diferentes niveles de altura en el Perú. Rev Per Ginecol Obstet. 2011;57(3):144-51.

3. Moore LG, Shelton MC, Colleen GJ. Humans at high altitude: hipoxya and fetal growth. Respir Physiol Neurobiol. 2011; 178(1):181-90. doi: 10.1016/j. resp.2011.04.017.

4. Yancey, Moore J, Brady K, Milligan D. Strampel W. The effect of altitude on umbilical cord blood gases. Obstet Gynecol. 1992;79(4):571-4.

5. Blickstein I, Green T. Umbilical Cord Blood Gases. Clin Perinatol. 2007;34(3):451-9.

6. Instituto Nacional de Estadística e Informática [Internet]. Lima, Perú: Densidad poblacional y altitud, según provincia, 2012 [citado 30 de octubre del 2012]. Disponible en http://www. inei.gob.pe/
7. Villamonte-Calanche W, Jerí-Palomino ME. Valores normales de peso al nacer a $3400 \mathrm{~m}$ de altura. Rev Per Ginecol Obstet. 2011;57(3):139-43.

8. Arikan GM, Scholz HS, Petru E, Haeusler MC, Haas J, Weiss PA. Cord blood oxygen saturation in vigorous infants at birth: what is normal? BJOG. 2000;107(8):987-94.

9. Link G, Clark K, Lang U. Umbilical blood flow during pregnancy: evidence for decreasing placental perfusion. Am J Obstet Gynecol 2007;196(5):489.e1-7.

10. Helwig JT, Parer JT, Kilpatrick SJ, Laros RK Jr. Umbilical cord blood acid-base state: What is normal? Am J Obstet Gynecol. 1996;174(6):1807-12.

11. WardropCAJ,HollandBM,VealeKEA, Jones JG, Gray OA. Nonphysyological anemia of prematurity. Arch Dis Chil. 1978;53(11):855-60.

12. Postigo L, Heredia G, Illsley NP, Torricos T, Dolan C, Echalar L, et al. Where the $\mathrm{O}_{2}$ goes to: preservation of human fetal oxygen delivery and consumption at high altitude. J Physiol. 2009; 587(Pt 3):693-708. doi: 10.1113/jphysiol.2008.163634.
13. Villamonte W, Jerí M. Crecimiento Fetal y peso al nacer en la altura [carta]. Rev Peru Med Exp Salud Publica. 2013;30(1):153-4.

14. Villamonte W, Jerí M, De la Torre C. Biometría fetal de 13 a 41 semanas a $3400 \mathrm{msnm}$ y su comparación con otros niveles de altura. Acta Med Per. 2013;30(1):14-25.

15. Villamonte W, Malaver J, Salinas R, Quispe E, Laurent A, Jerí $\mathrm{M}$ et al. Factores de los padres condicionantes del peso al nacer en recién nacidos a término a $3400 \mathrm{msnm}$. Rev Per Ginecol Obstet. 2011;157:153-63.

Correspondencia: Wilfredo Villamonte: Dirección: CENIMFA Urb. Villa del Carmen B4 San Jerónimo, Cusco.

Teléfono: 984930039

Correo electrónico: villamonte100@hotmail.com

\section{Consulte las ediciones anteriores de la Revista Peruana de Medicina Experimental y Salud Pública en} wWw.scielosp.org

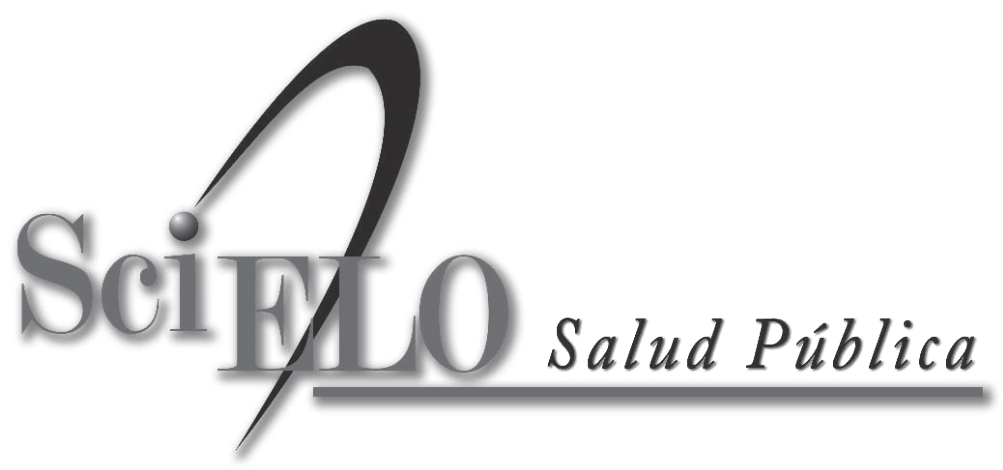

